\title{
DEAD TO THE WORLD: EMBODIED GENDER TRANSGRESSION AND THE LOSS OF HUMANITY
}

\author{
By Kerstyl C. Lowrey (New York University)
}

The accepted scope of humanity, though broad on first glance, actually represents a narrow, shallow stream. Within Western culture, the expectations, proscriptions and limitations tied to one's own "human" status remain largely unidentified until they are transgressed. The human body (lynchpin of humanity) is considered something not to be tampered with, at risk of the loss of one's human status. While some forms of modification, such as plastic surgery, tattooing, and piercing have become more broadly accepted, more extreme modifications of the body, intentional or otherwise, can render the subject as a freak - an implicit social death. Here emerges a curious overlap between the freak and the spectre, both inhabiting a liminal space between inhuman and exhuman, hypervisible in their distance from humanity. Broadly conceived of as mutilated or desecrated, transgender bodies are alternately read as cultural freaks and social specters, demonstrating the loss of humanity that occurs when transgression is physically written onto the body.

Transgender inhumanity emerges partially due to the ambiguous relationship between the trans body and death or spectrality. While the concept of "past lives" or reincarnation appears in many cultures, it is controlled by a literal understanding of death, which divorces humanity from the soul/spirit/etc. upon dying. However, past lives need not be limited to experiences bridging biological death. Generally, as an individual matures and develops, her identity shifts, leading to the creation of new "selves" (new lives, essentially), as previous selves/lives fade into the background. Instead of completely vanishing, these past selves become spectral, capable of reappearing to haunt 
current realities and lived experiences. Though past lives can be found haunting any individual, they become most apparent in cases of identity shifts against categories which are thought to be intransigent; cases of gender transition exemplify such past life hauntings. For transgender individuals, past genders ghost on the body, such that "organized forces and systemic structures that feel removed... make their impact felt in everyday life in a way that confounds... analytic... and social separations" (Gordon 2008: 19). In other words, the structures of the binary gender system become more apparent as they ghost through the experiences of transgender individuals. Gender haunts the transgender body, ghosting back and forth between present and previous lived genders. Rather than merely revealing the constructed (human?) nature of this binary, this transgressive gender ghosting serves to generate and structure transgender losses of humanity.

Ceridwen Troy once declared, "it is very easy to kill a transperson" (Troy 2008). In a compelling essay, she lists various discriminations and restrictions placed on visibly transgender bodies and subjects, ultimately concluding "[There] is a message hidden inside every single one of those statements: 'You are less than I am. You are not worthy of the rights and respect that I am worthy of. You are not human.' It is very easy to kill something that you do not see as human" (Troy 2008). Implicit throughout the essay is the oppressive force of the cultural assumption that, in terms of identity categories, the "ideal" transsexual ${ }^{1}$ vanishes. The only time that the transsexual should be visible, if at all, is during a phase of gender transition, a temporary freak process which involves the messy business of shifting gender presentation and adopting new, gender-appropriate behaviors. After this point, it is expected that she ${ }^{2}$ will fade into the world of normative gender, happily reassigned into a less dysphoric gender role. If not, as Troy observes, she 
is made invisible by more violently permanent means. This emphasis on transition and assimilation renders the transsexual experience as a sort of ghostly apparition, in which continued presence is ultimately determined by absence and invisibility. Though this invisibility is frequently chosen (whether freely or in a conscious endeavor to retain some scrap of humanity), it still must negotiate the ways in which "visibility is a complex system of permission and prohibition, presence and absence, punctuated alternately by apparitions and hysterical blindness" (Kipnis 1988: 158). The "hysterical blindness" which transsexual experiences mandate must be punctuated by apparitions of previous genders, for invisibility is only possible in the context of suppressing a (previously) visible entity ${ }^{3}$. Regardless of intentional invisibility, containing or limiting the haunting of (differently) gendered past lives is practically impossible.

The desire (or possibility) of eventual invisibility assumes a binary gender transition, from the socially accepted category of "male" or "female" to the other. For transgender individuals who never undergo a "full" (surgical/hormonal) transition, or who choose not to hide their transgender status, the haunting interplay of visibility and invisibility becomes more complex. Retaining visible gender transgression renders an individual as "the specter of the freak against which the norm is installed" (Butler 2001: 631). To remain in a transitional state, in between accepted gender categories, is to remain as an apparition, never fully observable within social codes of visibility. In other words, to remain the freak is to remain inextricably the Other.

Hegel's exploration of the dialectic between the self and the negating Other illuminates transgender inhumanity. The insistent stability of normative gender requires an Other in gender flux, performed in quotidian and framed contexts. Current contemporary imaginings of transgender bodies still partially locate it in the bearded 
ladies and "half-man-half-woman" freaks of nineteenth century sideshows. Regarding the power of the such liminal performances, Leslie Fiedler notes, "we perceive the 'freak' as the Other, the dark and unnatural mirror of ourselves" ( Fiedler 1978: 369). Audiences would not be so fascinated by the half-and-half if they could not on some level relate to it. On some level, society must feel slightly insecure in the realm of sex and gender in order to appreciate the practical paradox of a half-and half. The power of "half-and-half" performance, I would argue, is rooted in the seeming intransigence of biological sex. Human society firmly maintains that the human species is wholly sexually dimorphic, and any divergence from this "natural order" of binary opposition is rendered irrevocably Other. The performance of half-and-halves and bearded ladies served to enforce this construction of two binary sex categories; a conception of "normal" cannot exist without the existence of a marginal abnormality. Half-and-halves performed the necessary Other to shore up the inadequacies of the gender system, reassuring audiences in their own gender roles. Contemporary constructions of transgender bodies as negations of humanity perform a similar normalizing role.

Any instance of gender transgression can be read as a sort of social death. Describing her own transition, Kate Bornstein comments, “[g]ender enlightenment begins with death... I died a virtual death, not only on the operating table, but in terms of a key aspect of my identity, and then I was reborn into the world" (Bornstein 1994: 93-4). Social life necessitates a death in the case of gender transition because gender assignments are assumed to be rigid; rupturing the solidity of assigned categories mandates an exit from the social world into a liminal and interstitial realm. The exile of gender transgression from mainstream culture indicates its haunting potential, as Derrida notes that "hegemony still organizes repression and thus the confirmation of every 
haunting. Haunting belongs to the structure of every hegemony" (Derrida 1994: 46). The overwhelming prevalence of gender expectations and norms makes possible the haunting of transgender past lives, both for the individual and for her community. If the categories actually were stable, gender would not house so many ghosts. Because "identity categories are imaginary projections of an idealized wholeness that encrypts the mimetic and living transmissions of cultural memory," gender becomes a veritable field of freaks, specters and (not quite) forgotten apparitions (Rayner 2006: xxxi). These ghostly fragments of previous gender identities become reanimated as past lives, never fully disassociated from the bodies which they previously inhabited.

Perhaps the death that accompanies gender transgression/transition can be seen as a true case of "learning to live, finally," a lesson that can only be learned "between life and death" (Derrida 1994: xvii). Bornstein's "gender enlightenment" indicates a way of learning to live in a new way, an appropriation and reassessment of body and identity for one's own power, enjoyment, and comfort (Bornstein 1994: 93). The common transgender narrative that declares "I was trapped in the wrong body," or, "I wasn't living my life until I transitioned," encapsulates the possibility that gender transition can signify learning to live one's own life, “alone, from oneself" (Derrida 1994: xvii). Learning to live, finally, might even open the possibility of learning to live outside of the (dis)comforting structures of the hegemonic gender binary. In order for the transgender or transsexual individual to learn to live, finally, she must participate in the death of her former identity, which then reiterates as a past life throughout her continuing lived experiences. Regardless of the abuse predicated on transgender transgression, perhaps the freakish status which this "learning to live, finally" enables allows the newly inhuman 
transgender individual to exist outside of, or recreate, that which could be considered real.

Differently gendered past lives are written on the body. Surgical scars represent perhaps the most visible ghosts of previous genders, lingering from chest augmentations and reductions or from genital reconstruction surgeries. Scars reveal the refusal of the "hermetically sealed body" that human norms require, demonstrating that bodies can be cut open and reassembled, can "die a virtual death" and remain living (Peterson 2007: 129; Bornstein 1994: 94). Furthermore, the scar itself is spectral, decreasing in visibility with time; the scar is not the thing itself, but the trace of a previous feature and the event of its loss (or acquisition). In the words of Alice Rayner, "[ $t]$ he body is the memory of what is otherwise forgotten," and scars enact "[s]ites of distortion that betray the presence of unseen bodies" (Rayner 2006: 45, 69). Gender scars trigger the imagination and memory of the embodiment of past genders, engaging phantom bodies on the surface of present incarnations. The body projects the ghosts of its previous genders.

If scars represent the spectral remnants of past lives, then hormones indicate an ongoing attempt to exorcise the markers of previous genders that linger on the body. The work of banishing such an apparition (a strategy for the retention of humanity which is doomed to fail) requires continued engagement with gender ghosts, monitoring gender cues to create and maintain the desired (normative, non-freakish) gender presentation; the irony that hormonal and surgical intervention constitutes mutilation in some mainstream eyes bears note. Kessler and Mckenna observe that it takes at least four "female" gender cues to counteract one "male" cue, suggesting the difficulty of total and continued exorcism of past genders (Kessler and Mckenna 1978: 152). Conversely, an individual's current gender is socially attributed on the basis of her presumed "cultural genitals," an 
imagined apparition between their legs which matches the gender categories that the rest of her physical presentation suggests (Kessler and McKenna 1978: 154). Imagined genitalia haunt the body, attempting to regulate the alignment between presentation and embodiment. Though previous genders are physically embodied, their ghosting is a social phenomenon. The ways that physical gender transgression appears on the body triggers the invalidation, discrimination, and violence plaguing those unprotected by the bounds of "human decency."

Even if the specter of the freak is exorcized with a normative, 'passing' gender presentation, transgender past lives haunt social relationships as well. In the postmodern reality of constant surveillance and mediation, gender histories remain traceable in court documents, hospital records, and birth certificates ${ }^{4}$. The past life paper trail, however, does not have nearly the visceral impact of gender histories relating to family and other long-term human relationships. Here, the ghost floats off of the body and into the interstitial space between individuals, complicating their relationship due to their own individual apprehensions, expectations, and memories relating to the transgender past (and present) lives. A mother, learning that her once-daughter is now her son, may have difficulty letting go of her daughter's ghost, in order to welcome and support the new identity. The mother's remembrance of the daughter's humanity may cost the son his.

Transgender past lives become more embedded by some psychiatric rhetorics that encourage friends and family members to conceptualize of a gender transition as the death of their loved one, allowing for the reemergence of a new (similar, but not the same) relationship with the reborn individual, as if killing the "freak" allows for a preservation of stability. Such a mortal strategy was deployed by John Money in the infamous John/Joan case, in which he insisted that the parents think of their son as dead 
because "harboring doubts [could] weaken the child's [gender] identification" (Colapinto 2000: 54$)^{5}$. Instructions to envision death in association with transition structures the ghosting of gender in social interactions, allowing previous identifications to hover on a spectral periphery while their presence is consciously ignored or denied. Social gender hauntings reflect back onto continued gender embodiment, as "the relation of each body to itself is always haunted by its appropriation in and through the other" (Peterson 2007: 114). Gender specters live in and on the body, but they are sustained by social relationships; the persistence of past lives written on the body assumes other bodies to read them. Without social interactions, gendered past lives are confined to the realm of (the transgender individual's) memory.

Gender ghosts perform the haunting of transgender bodies regardless of one's acknowledgement or denial of past lives. Even if an individual "outs" herself as transgender or transsexual, her past as a different gender remains ghostly for new acquaintances. Rigidity of gender categories limits the imagination of previous embodied genders, only allowing for spectral traces. This specter, "understood as neither spirit nor body, but as a surplus," may emerge in physical characteristics that overflow current gender presentation—scars, or "oversized" hands ${ }^{6}-$-or may appear in memory and social interaction, conjuring the freakishness which underwrites transgender inhumanity (Peterson 2007: 66). Further, the specter projects forward, as future possible gender presentations haunt the current body and the social imagination; this remains particularly apparent in the case of the actively transitioning body, in a state of flux which may or may not target a specific destination. Transgender past lives are insensible to linear time, like Derrida's description of the specter which "is the future, it is always to come, it presents itself as only that which could come or could come back" (Derrida 1994: 48). 
Even in new social interactions, the ghosts of differently gendered past lives can never be fully exorcised; as specters, they are always in the process of reappearing. The danger of this re-apparition suggests that for the transgender subject, reclamation of human status may always already be a failed project.

Cultural scripts which render transgender bodies as freaks and specters release these subjects into the realm of the inhuman, revealing gaps and fissures in the structuring of humanity. The image of the freakishly gendered body, physical transgression writ large, socially justifies the negation of the humanity of trans subjects. Transgender past lives demonstrate the ways in which gender haunts and the body and social interactions. Such ghosting is not limited to gender-transgressive bodies, however; gender can be found haunting any individual, in the forms of expectations and structures. "Failing to live up to gender norms," to use Judith Butler's phrase, constitutes the root of any gender haunting, whether the failure comes prior to the haunting or results from it (Butler 2001: 634). Playing and performing through cultural structures, gender acts as a social apparition that ghosts, haunts, and ultimately possesses the body. In acknowledging, troubling, and subverting this possession, the inhumanly transgender subject accesses the privileges and dangers which exist outside of human decency. 


\section{Notes}

${ }^{1}$ For the purposes of this paper, I use "transsexual" to refer to individuals who undergo hormonal and surgical gender transition with the intention of living a 'stealth' life after transition, in which they are not out about their transsexual status. Conversely, I use "transgender" as an umbrella term including individuals who identify with a gender other than their assigned gender, but may or may not undergo surgical and/or hormonal transition.

${ }^{2}$ Feminist consciousness leads me to use she/her as a generic pronoun in this paper. This choice is not intended to silence, ignore, or obscure transmasculine experiences and subjectivities.

${ }^{3}$ This division of visible/invisible is an oversimplification, considering that past genders are always already potentially emergent. Building on Judith Butler's work which suggests that gender is an accomplishment, the possibility of gender haunting the body proposes that gender is never (completely) accomplished.

${ }^{4}$ Though it is possible for some of these records to be sealed or altered to comply with current gender identification and presentation, it is essentially impossible to erase all official traces of a differently gendered past. A transsexual can only be as 'stealth' as their own omission allows.

${ }^{5}$ Though Dr. Money's work on gender reassignment has been widely discredited by David Reimer himself (the subject of the John/Joan experiment), as well as scientists such as Milton Diamond and Keith Sigmundson, conflations of gender reassignment with death remains a commonly deployed coping strategy for families and friends coping with a loved one's transition.

${ }^{6}$ Large hands are often listed as a marker of male-to-female transsexuals, and represent a feature of the body which (currently) cannot be reshaped to gender congruence by surgery. 


\section{Works Cited}

Bornstein, Kate. Gender Outlaw: On Men, Women, and the Rest of Us. New York: Vintage Books, 1994.

Butler, Judith. "Doing Justice to Someone: Sex Reassignment and Allegories of Transsexuality." GLQ 7.4 (2001): 621-36.

Colapinto, John. As Nature Made Him: The Boy Who Was Raised as a Girl. New York: HarperCollins, 2000.

Derrida, Jacques. Specters of Marx: The State of the Debt, the Work of Mourning, and the New International. New York: Routledge, 1994.

Fiedler, Leslie. Freaks: Myths and Images of the Secret Self. New York: Anchor Books, 1978.

Gordon, Avery. Ghostly Matters: Haunting and the Sociological Imagination. Minneapolis: University of Minnesota Press, 2008.

Kessler, Suzanne J., and Wendy McKenna. Gender: An Ethnomethodological Approach. Chicago: University of Chicago Press, 1978.

Kipnis, Laura. "Feminism: The Political Conscience of Postmodernism?" Universal Abandon? The Politics of Postmodernism. Ed. Andrew Ross. Minneapolis: University of Minnesota Press, 1988. 149-66.

Peterson, Christopher. Kindred Specters: Death, Mourning, and American Affinity. Minneapolis: University of Minnesota Press, 2007.

Rayner, Alice. Ghosts: Death's Double and the Phenomena of Theatre. Minneapolis: University of Minnesota Press, 2006.

Troy, Ceridwen. "How to Kill a Transperson." The Empty Closet (March 2008), 
http://www.gayalliance.org/index.php?option $=$ com_mojo\&Itemid $=114 \& p=2205$

[accessed 25 September 2008]. 